\title{
Duty cycle of the radio galaxy B2 0258+35
}

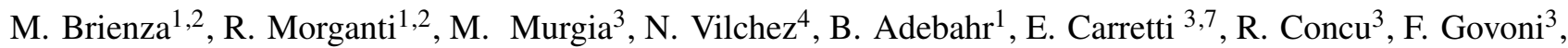

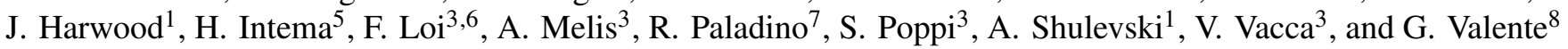 \\ 1 ASTRON, The Netherlands Institute for Radio Astronomy, Postbus 2, 7990 AA Dwingeloo, The Netherlands \\ e-mail: m.brienza@ira.inaf.it \\ 2 Kapteyn Astronomical Institute, Rijksuniversiteit Groningen, Landleven 12, 9747 AD Groningen, The Netherlands \\ 3 INAF - Osservatorio Astronomico di Cagliari, Via della Scienza 5, 09047 Selargius (CA), Italy \\ ${ }^{4}$ Qatar Environment and Energy Research Institute (QEERI), HBKU, Qatar Foundation, PO Box 5825, Doha, Qatar \\ 5 Leiden Observatory, Leiden University, Niels Bohrweg 2, 2333 CA Leiden, The Netherlands \\ ${ }^{6}$ Dipartimento di Fisica, University of Cagliari, Strada Prov.le Monserrato-Sestu Km 0.700, 09042 Monserrato (CA), Italy \\ 7 INAF - Istituto di Radioastronomia, Via Piero Gobetti 101, 40129 Bologna, Italy \\ 8 Agenzia Spaziale Italiana (ASI), Roma, Italy
}

Received 17 February 2018 / Accepted 16 July 2018

\begin{abstract}
Context. Radio loud active galactic nuclei (AGN) are episodic in nature, cycling through periods of activity and quiescence. The study of this duty cycle has recently gained new relevance because of the importance of AGN feedback for galaxy evolution.

Aims. In this work we investigate the duty cycle of the radio galaxy B2 $0258+35$, which was previously suggested to be a restarted radio galaxy based on its morphology. The radio source consists of a pair of kpc-scale jets embedded in two large-scale lobes $(\sim 240 \mathrm{kpc})$ with relaxed shape and very low surface brightness, which resemble remnants of a past AGN activity.

Methods. We have combined new LOFAR data at $145 \mathrm{MHz}$ and new Sardinia Radio Telescope data at $6600 \mathrm{MHz}$ with available WSRT data at $1400 \mathrm{MHz}$ to investigate the spectral properties of the outer lobes and derive their age.

Results. Interestingly, the spectrum of both the outer northern and southern lobes is not ultra-steep as expected for an old ageing plasma with spectral index values equal to $\alpha_{1400}^{145}=0.48 \pm 0.11$ and $\alpha_{6600}^{1400}=0.69 \pm 0.20$ in the outer northern lobe, and $\alpha_{1400}^{145}=0.73 \pm 0.07$ in the outer southern lobe. Moreover, despite the wide frequency coverage available for the outer northern lobe $(145-6600 \mathrm{MHz})$, we do not identify a significant spectral curvature $(\mathrm{SPC} \simeq 0.2 \pm 0.2)$.

Conclusions. While mechanisms such as in-situ particle reacceleration, mixing or compression can temporarily play a role in preventing the spectrum from steepening, in no case seem the outer lobes to be compatible with being very old remnants of past activity as previously suggested (with age $\gtrsim 80 \mathrm{Myr}$ ). We conclude that either the large-scale lobes are still fuelled by the nuclear engine or the jets have switched off no more than a few tens of Myr ago, allowing us to observe both the inner and outer structure simultaneously. Our study shows the importance of combining morphological and spectral properties to reliably classify the evolutionary stage of low surface brightness, diffuse emission that low frequency observations are revealing around a growing number of radio sources.
\end{abstract}

Key words. radio continuum: galaxies - galaxies: jets - galaxies: individual: NGC 1167 - galaxies: individual: B2 0258+35

\section{Introduction}

Some of the outstanding questions in the study of radioloud active galactic nuclei (AGN) are concerned with how long and how frequently they are active, and how to identify the various phases of their evolution. Statistical studies and models suggest that the AGN duty cycle strongly depends on the power of the radio source (Best et al. 2005; Shabala et al. 2008; Turner \& Shabala 2015) but precise estimates are still not available. Understanding the life cycle of AGN jets has recently gained a new broader relevance in the context of radio galaxy evolution (Morganti 2017), as they can transfer a significant amount of energy into the ambient medium (McNamara \& Nulsen 2007; Wagner \& Bicknell 2011; Wagner et al. 2012; Gaspari et al. 2012). Feedback from AGN is indeed required by all cosmological simulations to explain the quenching of the star formation in early type galaxies and the correlation between galaxy, and black hole properties (Di Matteo et al. 2005; Fabian 2012; Schaye et al. 2015; Sijacki et al. 2015).
Radio observations are particularly suited to trace the different stages of evolution in radio galaxies via the study of their radio morphology and spectra. Evidence of radio galaxies in different phases of their evolution has been presented in the literature as described below.

For example, remnant radio galaxies represent the last phase when the jets have switched off (e.g. Parma et al. 2007; Murgia et al. 2011; Saripalli et al. 2012). Recent studies based on low frequency observations and modelling of radio galaxies find fractions of remnants $\lesssim 10-15 \%$, suggesting a rapid expansion of the plasma in the ambient medium after the nuclear activity has ceased (Godfrey et al. 2017; Brienza et al. 2017; Mahatma et al. 2018). Observations show that remnants can have different characteristics, probably depending on their stage of evolution. At $\mathrm{GHz}$ frequencies they are commonly observed to have ultra-steep spectra ( $\mathrm{S} \propto v^{-\alpha}, \alpha>1.2$, e.g. Parma et al. 2007; Hurley-Walker et al. 2015; Shulevski et al. 2017), typical of old ageing plasma, but can also have only moderately steep spectra at $\mathrm{MHz}$ frequencies $(0.6<\alpha<1$, e.g. Murgia et al. 2011; Brienza et al. 2016; Mahatma et al. 2018). Moreover, they 
can show very weak radio cores indicating that the nuclear activity may not completely switch off but just go through a period of significant suppression (Murgia et al. 2011; Brienza et al. 2016, 2017; Mahatma et al. 2018).

Sources that exhibit remnant emitting plasma from past activity and, at the same time, new-born jets are named restarted radio galaxies (see Saikia \& Jamrozy 2009, for a review). These can be effectively used to investigate the duty cycle of the radio AGN.

The most explicit and well-known signatures of recurrent jet activity on large scales are "double-double radio galaxies" (DDRGs). This class of objects was first defined by Schoenmakers et al. (2000) as "radio galaxies consisting of a pair of double radio sources with a common centre" and further investigated by other authors (e.g. Kaiser et al. 2000; Konar \& Hardcastle 2013; Orrù et al. 2015). For these objects the duration of the quiescent phase between two episodes of jet activity is estimated to be in the range $10^{5}-10^{7} \mathrm{yr}$, and is found to be typically shorter than the duration of the previous active phase equal to $10^{8} \mathrm{yr}$ on average (Konar et al. 2013). However, the phenomenon of restarted AGN is not limited to DDRGs. Another class of sources is observed to have compact inner jets embedded in large-scale, low-surface brightness lobes (e.g. Jamrozy et al. 2009; Kuźmicz et al. 2017). In some of these sources, there are indications that the quiescent phase may be as long as $\geq 10^{8} \mathrm{yr}$ (Jamrozy et al. 2007). Among the most famous cases of restarted jets is the radio galaxy Centaurus $A$, where more than one phase of activity has been claimed based on the presence of two distinct morphology structures (Morganti et al. 1999; McKinley et al. 2013, 2018). Moreover, observations of remnant emission on small scales $(<100 \mathrm{pc})$ associated to active compact radio galaxies (Luo et al. 2007; Orienti \& Dallacasa 2008) suggest that at the beginning of the jet activity, multiple cycles of short bursts $\left(10^{3}-10^{4} \mathrm{yr}\right)$ may occur before the jets start to expand to large scales.

The physical mechanisms that drive the radio jets intermittence are still poorly understood and are possibly related to the galaxy environment. Some possible explanations include gas-rich galaxy-galaxy interactions (e.g. Schoenmakers et al. 2000), perturbations of the accretion process (e.g. Pringle 1997; Czerny et al. 2009; Wu 2009) and chaotic cold accretion (e.g. Gaspari et al. 2013, 2017). Alternative interpretations to the restarting scenario have also been proposed to explain the observed morphologies of these sources. For example, Baum et al. (1990) suggest that compact radio sources can arise within a large-scale radio galaxy if the jet propagation is obstructed or impeded on scales of tens of parsecs to few kiloparsecs. In that case, the extended structure would remain visible along with the confined parsec scale source.

The variety of sources described above suggests that a multitude of situations and evolution histories can occur. As a consequence, the interpretation and classification of restarted sources is not trivial and more examples need to be studied in detail for improving our understanding. In particular, extra constraints can be obtained by studying the spectral properties of these sources on a broad spectral range, from $\mathrm{MHz}$ to $\mathrm{GHz}$ frequencies, in combination with the source morphology. A better characterization of known restarted radio galaxies is also essential for a better selection and census of restarted sources in upcoming large-area radio surveys (e.g. the LOw Frequency ARray (LOFAR) Twometre Sky Survey - LoTSS, Shimwell et al. 2017).

In this paper we present new observations of the radio source B2 0258+35, where two low-surface brightness extended lobes $(240 \mathrm{kpc}$ ) have been discovered at $1400 \mathrm{MHz}$ (Shulevski et al.
2012) around a compact radio galaxy $(3 \mathrm{kpc})$. Due to its morphology, this source has been interpreted as a restarted radio galaxy in which the outer lobes represent old remnants of a past AGN activity. The aim of this work is to put new constraints on the physical properties and duty cycle of these lobes by studying their spectral properties and morphology over a wide frequency range.

The cosmology adopted in this work assumes a flat universe and the following parameters: $H_{0}=70 \mathrm{~km} \mathrm{~s}^{-1} \mathrm{Mpc}^{-1}$, $\Omega_{\lambda}=0.7, \Omega_{\mathrm{M}}=0.3$. At the redshift of $\mathrm{B} 20258+35$ equal to $z=0.0165$ (Wegner et al. 1993), 1 arcsec corresponds to $0.34 \mathrm{kpc}$. Throughout the paper the spectral index, $\alpha$, is defined using the convention $S \propto v^{\alpha}$.

\section{Overview on the source B2 $0258+35$}

The radio source B2 $0258+35$ is hosted by the giant early-type galaxy NGC $1167\left(M_{\mathrm{B}}=-21.7 \mathrm{mag}, D_{25}=56 \mathrm{kpc}\right)$, which is optically classified as a Seyfert 2 galaxy (Ho et al. 1997). The galaxy is located at redshift $z=0.0165$ (Wegner et al. 1993) and does not belong to any galaxy group but is surrounded by a few smaller satellites (Struve et al. 2010). Deep optical observations show a faint, tightly wound spiral structure at $r<30 \mathrm{kpc}$, which represents the signature of a past major merger event (Emonts et al. 2010).

Indications of past and/or ongoing interactions are also confirmed by the presence and kinematics of a large $(\sim 160 \mathrm{kpc})$ and massive $\mathrm{HI}$ disk $\left(M_{\mathrm{HI}}=1.5 \times 10^{10} M_{\odot}\right.$, Noordermeer et al. 2005, Emonts et al. 2010, Struve et al. 2010). This HI structure is thought to have assembled via accretion of gas-rich satellite galaxies. However, the gas distribution is extremely regular out to $65 \mathrm{kpc}$, with disturbed kinematics only in the very outer part, which is possibly the result of recent interactions with its satellites. Thanks to its inner regular kinematics, Struve et al. (2010) have computed a tight lower limit on the epoch of the last major merger event equal to $\sim 1 \mathrm{Gyr}$. This timescale is also supported by the absence of young stellar population signatures in the optical spectrum (Emonts 2006). However, more recent interactions with small companion galaxies cannot be ruled out.

$\mathrm{HI}$ is also observed in absorption against the the central radio source (Emonts et al. 2010; Murthy et al., in prep.). The broad absorption profile is likely the result of the combination of a circumnuclear disk disturbed by the interaction with the radio jet (Murthy et al., in prep.). Support for an on-going interaction also comes from the molecular gas (Murthy et al., in prep. using observations from Prandoni et al. 2007). Surprisingly, there is no evidence of broadening in the optical emission lines of the ionized gas (Emonts 2006; Santoro, priv. comm.).

The central radio source has a luminosity at $408 \mathrm{MHz}$ of $L_{408}=10^{24.37} \mathrm{~W} \mathrm{~Hz}^{-1}$ and has been classified as a Compact Steep Spectrum (CSS) source by Sanghera et al. (1995). As shown in the inset in Fig. 1, sub-arsecond imaging shows two plum-like lobes of $\sim 3 \mathrm{kpc}$ total projectd size, with jetlike structures but without clear hot-spots (Giroletti et al. 2005; Giovannini et al. 2001). From the jet to counter-jet ratio at $22000 \mathrm{MHz}$, Giroletti et al. (2005) have constrained the jets inclination angle to a value in the range $40 \mathrm{deg}<\theta<50 \mathrm{deg}$, which is in accordance with the optical classification of the parent galaxy. Using radiative evolution models they have derived a source age of $0.9 \mathrm{Myr}$ and, based on dynamical arguments, have suggest that the radio source might not grow into an extended FRI/II radio galaxy. This is further supported by the clear bending of the southern lobe indicating a dense surrounding interstellar medium (ISM), which may be the cause of the source 


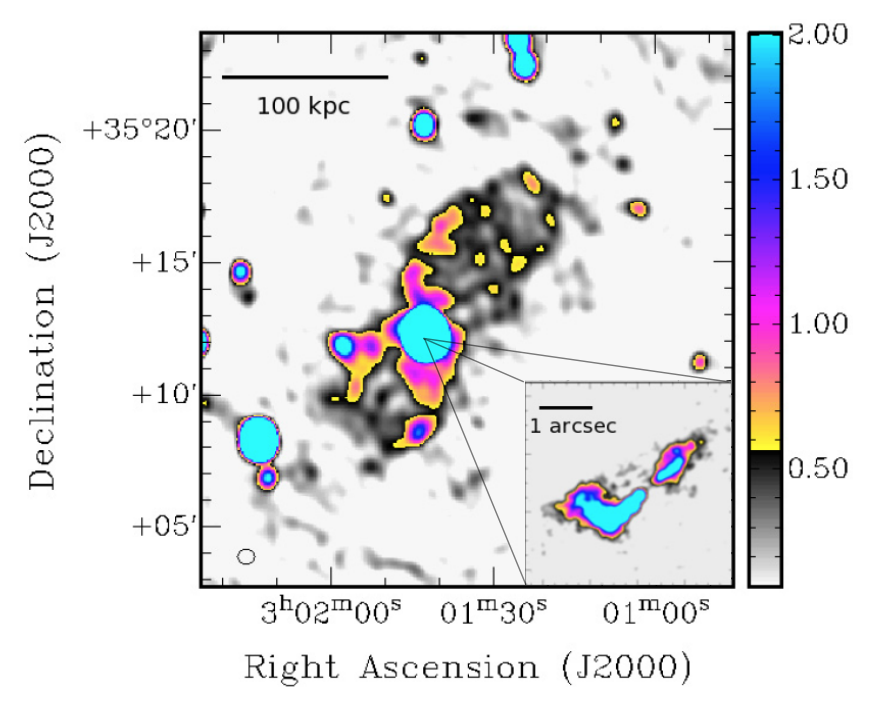

Fig. 1. Continuum image of the radio galaxy $\mathrm{B} 20258+35$ at $1400 \mathrm{MHz}$ obtained with the Westerbork Synhesis Radio Telescope from Shulevski et al. (2012). The synthesized beam is indicated with the ellipse at bottom left. The intensity ranges from $100 \mu \mathrm{Jy}(1 \sigma)$ to $2 \mathrm{mJy}(20 \sigma)$. The inset at the bottom right shows a high-resolution radio image at $8400 \mathrm{MHz}$ of the central CSS source obtained with the Very Large Array by Giroletti et al. (2005).

confinement and is in agreement with the jet-ISM interaction inferred from HI absorption (Murthy et al., in prep.). The spectral index distribution within the CSS source varies from $\alpha_{8.4}^{22}=0$ in the core, to $\alpha_{8.4}^{22}=0.6$ in the jet-like structures within the lobes, to $\alpha_{8.4}^{22}=1.0-1.5$ in the surrounding diffuse emission (Giroletti et al. 2005).

A very interesting ingredient for understanding the evolutionary history of the source is the presence of two largescale $(240 \mathrm{kpc})$, low-surface brightness $\left(1.4 \mathrm{mJy} \mathrm{arcmin}^{-2}\right.$ at $1400 \mathrm{MHz}$ ) lobes that surround the CSS source. These have been first detected and studied at $1400 \mathrm{MHz}$ by Shulevski et al. (2012) using the Westerbork Synhesis Radio Telescope (WSRT; Fig. 1). The authors propose that the lobes represent old remnants of a previous cycle of jet activity. The S-shape morphology of the broad enhancements in surface brightness located at the edges of the outer lobes is also notable.

Assuming the lobes to be buoyant bubbles expanding in the intergalactic medium, Shulevski et al. (2012) estimate the dynamical age of the source to be $\gtrsim 80 \mathrm{Myr}$. Because the age of the young central CSS source is about $0.9 \mathrm{Myr}$, they suggest that the quiescent phase between the two radio bursts has lasted $\sim 100$ Myr.

The main characteristics of the radio source B2 $0258+35$ and of its host galaxy are summarised in Table 1.

\section{Data}

To investigate the radio spectral properties of the extended lobes and to constrain any spectral curvature, we expand here the study at $1400 \mathrm{MHz}$ presented by Shulevski et al. (2012) to other frequencies. We have performed observations at 145 , 350 and $6600 \mathrm{MHz}$ using the Low-frequency Array (LOFAR, van Haarlem et al. 2013), the Karl G. Jansky Very Large Array (VLA), and the Sardinia Radio Telescope (SRT, Bolli et al. 2015; Prandoni et al. 2017) respectively. Moreover, we have reprocessed archival observations of the Giant Metrewave Radio Telescope (GMRT; Swarup 1991) taken at 235 and $612 \mathrm{MHz}$.
Table 1. Summary of the properties of the radio source B2 $0258+35$ and of its host galaxy.

\begin{tabular}{cc}
\hline \hline Host galaxy & NGC 1167 \\
$M_{\mathrm{B}}$ & $-21.7 \mathrm{mag}$ \\
$D_{25}$ & $56 \mathrm{kpc}$ \\
Optical classification & Seyfert 2 \\
Redshift & 0.0165 \\
Size inner radio lobes & $3 \mathrm{kpc}$ \\
Inner lobes radio power & $L_{408}=10^{24.37} \mathrm{~W} \mathrm{~Hz}^{-1}$ \\
Size outer radio lobes & $240 \mathrm{kpc}$ \\
$M_{\mathrm{HI}}$ & $1.5 \times 10^{10} M_{\odot}$ \\
Diameter Hi disk & $160 \mathrm{kpc}$ \\
\hline
\end{tabular}

Unfortunately, due to the data quality and dynamic range limitations caused by the bright central source, we could not recover the outer lobe emission at 235, 350 and $612 \mathrm{MHz}$. However, these frequencies have been useful for the study of the compact source. For the analysis of the outer lobes we have also used the WSRT image at $1400 \mathrm{MHz}$ presented by Shulevski et al. (2012). In the following sections we describe the observations and the data reduction procedures. A summary of the observational details is presented in Table 2 and a summary of the final image properties is presented in Table 3.

\subsection{LOFAR HBA observations and data reduction}

Dedicated observations of the source were performed using the LOFAR High Band Antenna (HBA) on September 13th, 2015. All the 64 antennas of the Dutch Array have been used, providing a maximum baseline of $\sim 100 \mathrm{~km}$. The target was observed in scans of $30 \mathrm{~min}$ for a total integration time of $8 \mathrm{~h}$, interleaved by 2-min observations of the flux-density calibrator 3C48. The sampling time was set to $1 \mathrm{~s}$ and four polarization products (XX, YY, XY, and YX) were recorded. The total observed bandwidth is equal to $95.1 \mathrm{MHz}$ in the range $100.2-195.3 \mathrm{MHz}$. This was divided in 487 sub-bands of $195.3 \mathrm{kHz}$ composed of 64 channels each. The observational details are summarized in Table 2.

Using the observatory pipeline (Heald et al. 2010) the data were pre-processed. Data below $108.9 \mathrm{MHz}$ and above 176.9 $\mathrm{MHz}$ were flagged and excluded from further processing due to the presence of strong RFIs.

After pre-processing the data were calibrated using the classical direction-indipendent procedure (e.g. Brienza et al. 2016; Mahony et al. 2016). Amplitude and phase solutions for each station were calculated according to the model of $3 \mathrm{C} 48$ presented by Scaife \& Heald (2012) and transferred to the target field. The data were then combined into groups of ten subbands (corresponding to a bandwidth of $\sim 2 \mathrm{MHz}$ ) and phase self-calibrated iteratively, adding progressively longer baselines. The LOFAR imager AWImager (Tasse et al. 2013) was used for the imaging, which performs both w-projection to account for non-coplanar effects (Cornwell \& Perley 1992) and A-projection to account for the changing beam throughout the observation (Bhatnagar et al. 2008).

We produced images at different resolutions and with different weightings. The best detection of the outer lobes was achieved with a uv-cut equal to $2 \mathrm{k} \lambda$, robust weighting equal to 0 and final resolution of $80 \operatorname{arcsec} \times 98$ arcsec. Hints of the lobes emission are observed at higher resolution too but the image quality is not enough for a reliable detection and flux density measurement of the low surface brightness 
Table 2. Summary of the observational details.

\begin{tabular}{ccccccr}
\hline \hline Telescope & Configuration & $\begin{array}{c}\text { Frequencies } \\
\mathrm{MHz}\end{array}$ & $\begin{array}{c}\text { Target } \\
\text { TOS }(\mathrm{h})\end{array}$ & Calibrator & $\begin{array}{c}\text { Calibrator } \\
\text { TOS }(\mathrm{h})\end{array}$ & Observation date \\
\hline LOFAR & HBA Inner & $118.9-176.9$ & 8 & $3 \mathrm{C} 48$ & 0.53 & 13 September 2015 \\
GMRT $^{a}$ & - & 235 & 2.3 & $3 \mathrm{C} 48,0432+416$ & 0.75 & $24-25 \mathrm{July} 2011$ \\
VLA & CnB-B & $224-480$ & 2.25 & $3 \mathrm{C} 48$ & 0.5 & 6 February-10 April 2015 \\
GMRT $^{a}$ & - & 612 & 2.3 & $3 \mathrm{C} 48,0432+416$ & 0.75 & 24-25 July 2011 \\
SRT & - & $6000-7200$ & 11 & 3C286, 3C138 & 2 & 1-2-6-7 February 2016 \\
\hline
\end{tabular}

Notes. ${ }^{(a)}$ Archival observations.

Table 3. Summary of the image properties at different frequencies.

\begin{tabular}{ccc}
\hline \hline $\begin{array}{c}\text { Frequency } \\
\text { MHz }\end{array}$ & $\begin{array}{c}F W H M \\
\operatorname{arcsec}^{2}\end{array}$ & $\begin{array}{c}\text { RMS } \\
\text { mJy beam }^{-1}\end{array}$ \\
\hline 145 & $80 \times 98$ & 3 \\
235 & $9.3 \times 13.6$ & 1.2 \\
350 & $30 \times 30$ & 1.2 \\
612 & $3.6 \times 6.5$ & 0.2 \\
$1400^{a}$ & $33 \times 39$ & 0.1 \\
6600 & $174 \times 174$ & 0.9 \\
\hline
\end{tabular}

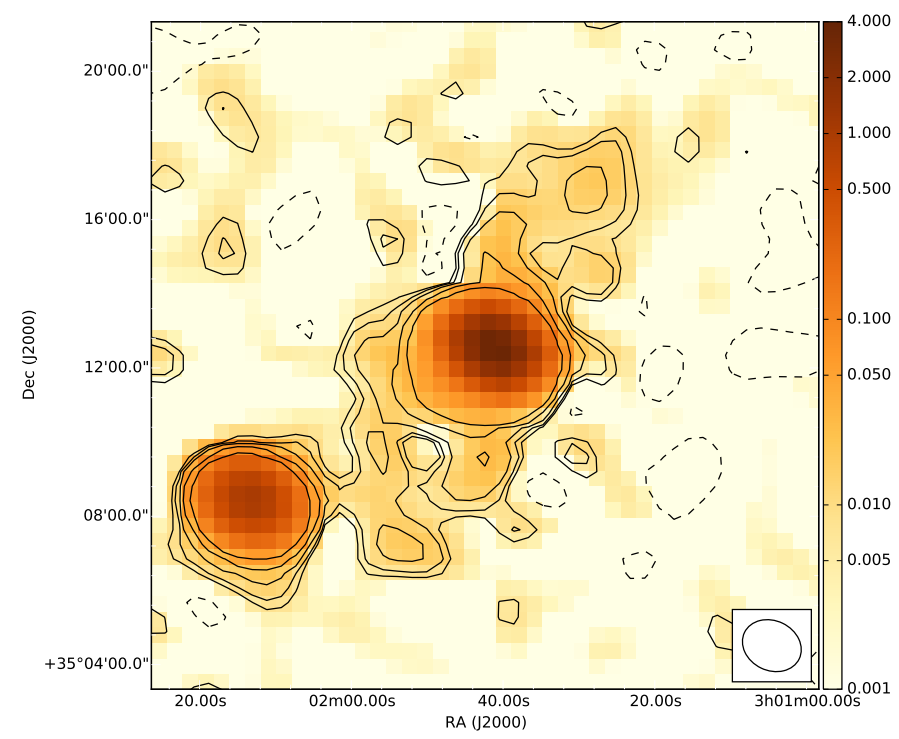

Notes. ${ }^{(a)}$ Image taken from Shulevski et al. (2012).

emission due to the presence of deconvolution artefacts associated with the strong central source. The calibration scheme created by van Weeren et al. (2016) and Williams et al. (2016), which corrects for direction-dependent errors due to ionospheric phase and amplitude distortions, did not provide any significant improvement of the dynamic range at resolutions higher than $80 \operatorname{arcsec} \times 98$ arcsec.

Therefore, in the following work we have used the image obtained with the original direction-independent calibrated data. The final image was obtained by combining all images at different frequencies in the image plane. It has a central frequency of $145 \mathrm{MHz}$, a spatial resolution of $80 \operatorname{arcsec} \times 98 \operatorname{arcsec}$ and a noise of $3 \mathrm{mJy} \mathrm{beam}^{-1}$ in the proximity of the target (see Fig. 2, top panel).

\subsection{SRT observations and data reduction}

The source was observed with the SRT on February 1-2-6-7, 2016 as part of the early science programme "SRT Multifrequency Observations of Galaxy Clusters" (SMOG - PI M. Murgia, see also Govoni et al. 2017; Loi et al. 2017; Vacca et al. 2018). A region of $0.7 \mathrm{deg} \times 0.7 \mathrm{deg}$ was mapped using the $C$-Band receiver for a total of $11 \mathrm{~h}$. We performed several on-thefly (OTF) mappings in the equatorial frame in both right ascension (RA) and declination (Dec). The FWHM of the beam at this frequency is 2.9 arcmin so we set the telescope scanning speed to 6 arcmin $\mathrm{s}^{-1}$ and the scan separation to 0.7 arcmin to properly sample the beam. Full Stokes parameters were recorded with the SARDARA backend (SArdinia Roach2-based Digital Architecture for Radio Astronomy; Murgia et al. 2016; Melis et al. 2018). The correlator configuration was set to 1024 frequency channels of approximately $1.46 \mathrm{MHz}$ for a total bandwidth of $1500 \mathrm{MHz}$. We then set the Local Oscillator to $5900 \mathrm{MHz}$ and we used a filter to select frequency range from 6000 to $7200 \mathrm{MHz}$, which is relatively free from strong RFIs. The central frequency is thus $6600 \mathrm{MHz}$ and the total bandwidth is $1200 \mathrm{MHz}$. A summary of the SRT observations is listed in Table 2.

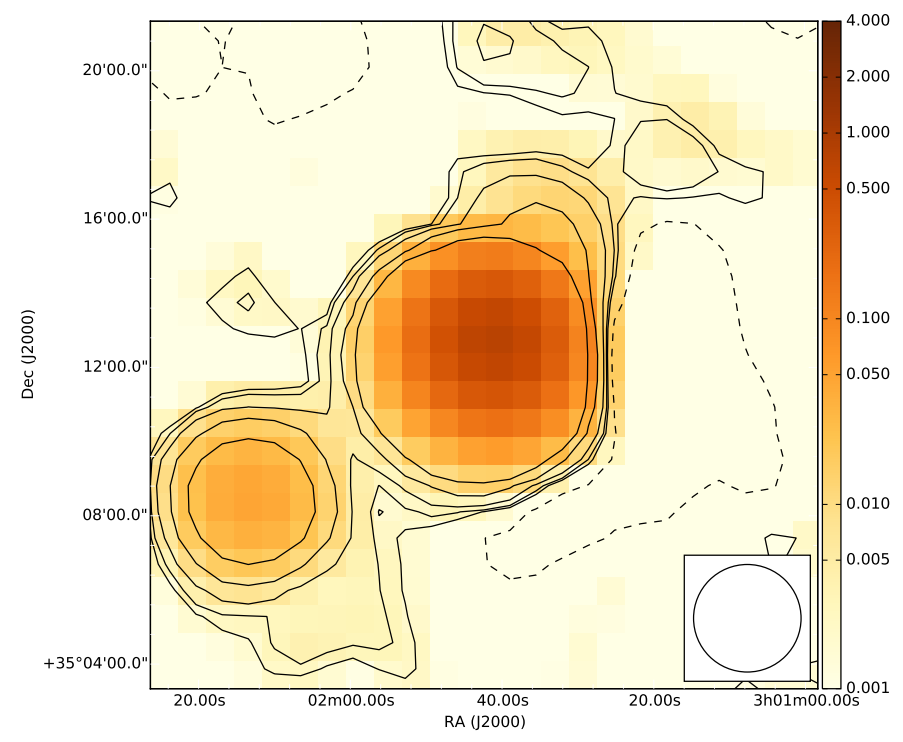

Fig. 2. Radio maps of the source B2 0258+35. Top panel: LOFAR 145MHz map at 80 arcsec $\times 98$ arcsec resolution. Levels: $-2,2,3,5,10,20$ $\times \sigma\left(3\right.$ mJy beam $\left.^{-1}\right)$. Bottom panel: SRT $6600-\mathrm{MHz}$ map at 2.9 -arcmin resolution. Levels: $-2,2,3,5,10,20 \times \sigma\left(0.9 \mathrm{mJy}_{\text {beam }}{ }^{-1}\right)$. The colour scale is set in Jy beam ${ }^{-1}$. In both panels the beam is shown at the bottom right corner.

After flagging RFIs, we performed the bandpass and fluxdensity calibration using observations of the sources 3C286 and 3C138. The flux density scale was set according to Perley \& Butler (2013). All data observed during February 7th, 
were completely discarded due to low quality resulting from bad weather conditions during the observing run. We processed the data using the proprietary Single-dish Spectral-polarimetry Software (SCUBE; Murgia et al. 2016). We applied the gainelevation curve correction to account for the gain variation with elevation caused by the telescope structure changes due to the gravitational stress.

We proceeded with fitting the baseline with a second order polynomial and subtracting it from each calibrated scan. To perform the imaging we projected the data in a regular threedimensional grid with a spatial resolution of $42 \mathrm{arcsec} \mathrm{pixel}^{-1}$ so that four pixels subtend the beam FWHM. All scans along the two orthogonal axes (RA and Dec) were then stacked together to produce the Stokes I image. In the combination, the individual image cubes were averaged and de-stripped by mixing their Stationary Wavelet Transform (SWT) coefficients as described in Murgia et al. (2016). We then used the higher signal-to-noise image cubes obtained from the SWT stacking as a prior model to refine the baseline fit.

Because the central source is very strong, a high dynamical range was required to be able to detect the extended, low surface brightness lobes in the central region of the map. To achieve this goal we deconvolved the sky image with a beam model pattern following Murgia et al. (2016) in order to remove the beam sidelobes. The deconvolution algorithm interactively finds the peak in the image obtained from the SWT stacking of all images and subtracts a fixed gain fraction (typically 0.1 ) of this point source flux convolved with the re-projected telescope "dirty beam model" from the individual images. In the re-projection, the exact elevation and parallactic angle for each pixel in the unstacked images are used. The residual images were stacked again and the CLEAN continued until convergence. As a final step, CLEAN components at the same position were merged, smoothed with a circular Gaussian beam with FWHM 2.9 arcmin, and then restored back in the residuals image to obtain a CLEANed image. The final image obtained in this way has a noise of $0.9 \mathrm{mJy}^{\text {beam }}{ }^{-1}$ and is shown in Fig. 2 (bottom panel).

\subsection{VLA P-band observations and data reduction}

We performed observations of the source with the VLA in two slots on February 6th, 2015 and April 10th, 2015 with the $P$-band receiver. On both runs, 27 antennas were used, distributed in CnB-B and B configuration in the two different runs respectively. The target and flux density calibrator 3C48 were observed for $1.5 \mathrm{~h}$ and $20 \mathrm{~min}$ on the first day and $45 \mathrm{~min}$ and ten minutes on the second day respectively. The sampling time was set to $2 \mathrm{~s}$ and four polarization products (RR, LL, RL, and LR) were recorded. The total bandwidth, equal to $256 \mathrm{MHz}$ in the range $224-480 \mathrm{MHz}$, was divided by default in 16 sub-bands of $16 \mathrm{MHz}$ with 128 frequency channels. The observational details are summarized in Table 2.

All datasets were reduced using the following steps. After having applied the flags suggested by the observatory, the data were flagged automatically using the AOFlagger, visually checked and further manually flagged when required. We used the Common Astronomy Software Applications (CASA, version 4.7, McMullin et al. 2007) to perform the calibration in the standard manner and following the guidelines set out in the online tutorial for continuum $P$-band data $^{1}$. The flux scale was

\footnotetext{
1 https://casaguides.nrao.edu/index.php/VLA_Radio_ galaxy_3C_129:_P-band_continuum_tutorial-CASA4.7.0
}

set according to Scaife \& Heald (2012). Phase and amplitude self-calibration was performed. The final image of the field is $2 \operatorname{deg} \times 2$ deg in size and was made using a Brigg's weighting with a robustness parameter of 0 . The image has a resolution of $30 \operatorname{arcsec} \times 30 \operatorname{arcsec}$ and central noise of $\sim 1.2 \mathrm{mJy}$ beam $^{-1}$. As already mentioned, due to severe flagging caused by the presence of extensive RFIs and the dynamic range limitations caused by the bright central source, the sensitivity of the data is not sufficient to detect the low surface brightness emission of the outer lobes of the radio galaxy.

\subsection{GMRT observations and data reduction}

We used archival data of the GMRT at 235 and $612 \mathrm{MHz}$. The observations were performed on July 24th and 25th, 2011. The target was observed in five time-scans for a total integration time of $2.3 \mathrm{~h}$. The source 3C48 was used as flux-density calibrator and observed at the beginning and at the end of the observing run for ten minutes respectively. We adopt the Scaife-Heald model (Scaife \& Heald 2012) for setting the absolute flux scale. Data at both frequencies were recorded simultaneously in singlepolarization mode using a 33-MHz bandwidth divided into 512 channels of $65-\mathrm{kHz}$ bandwidth and a sampling time of $16.1 \mathrm{~s}$.

We processed the data using the SPAM pipeline (Intema 2014; Intema et al. 2017). The output calibrated visibility data were imported into CASA to produce images at different resolutions. By using uniform weighting during imaging, we obtained a resolution of $3.6 \operatorname{arcsec} \times 6.5 \operatorname{arcsec}$ and $9.3 \operatorname{arcsec} \times 13.6 \operatorname{arcsec}$ in the $612-\mathrm{MHz}$ map and $235-\mathrm{MHz}$ maps, respectively. The noise is equal to $200 \mathrm{mJybeam}^{-1}$ at $612 \mathrm{MHz}$ and $1.2 \mathrm{mJy}^{-1}$ aeam ${ }^{-1}$ at $235 \mathrm{MHz}$. We also imaged the data using Briggs weighting with robust $=0$ and uvtaper $=40$ arcsec to enhance the large-scale emission. The final images with $\sim 40$ arcsec resolution have a noise levels equal to $\sim 1.2 \mathrm{mJy} \mathrm{beam}^{-1}$ and $12 \mathrm{mJy} \mathrm{beam}^{-1}$ at $612 \mathrm{MHz}$ and at $235 \mathrm{MHz}$, respectively. Unfortunately, with neither imaging weighting schemes we managed to detect the low surface brightness emission of the outer lobes of the radio galaxy.

\section{Results}

\subsection{Morphology}

In Fig. 2 we show the two radio continuum images of the source B2 $0258+35$ where the outer lobes are detected at $145 \mathrm{MHz}$ and $6600 \mathrm{MHz}$ respectively. As already mentioned in Sect. 2, the low surface brightness emission of the outer lobes has not been recovered at either $235 \mathrm{MHz}, 350 \mathrm{MHz}$ or $612 \mathrm{MHz}$, therefore we do not show those images here. We also note the presence of a point-like unrelated source located in south-east direction (RA 03:02:13.12, Dec +35:08:20.79, J2000).

In the LOFAR map both lobes are clearly visible. Their linear extension and morphology is in general agreement with what has been previously observed at $1400 \mathrm{MHz}(240 \mathrm{kpc}$, Shulevski et al. 2012). We measure a total angular extension of $\sim 13 \mathrm{arcmin}$, using the $3 \sigma$ contours at the tip of each lobe as a reference, which corresponds to a linear size equal to $\sim 265 \mathrm{kpc}$. The observed size difference with respect to Shulevski et al. (2012) may be partially attributed to the lower resolution of the LOFAR image and partially to an intrinsic larger extent of the source at low frequencies, especially of the southern lobe.

The total flux density of the outer lobes at $145 \mathrm{MHz}$ is $285 \mathrm{mJy}$ (obtained as the difference between the total flux density of the source and the peak flux density of the central 
component) and their average surface brightness is equal to $4.7 \mathrm{mJy} \mathrm{arcmin}^{-2}$. We note that the outer lobes represent only $\sim 6 \%$ of the entire radio emission of the source at $145 \mathrm{MHz}$.

Due to the lower angular resolution of the LOFAR map, the S-shape morphology that is clearly visible in the map at 1400 MHz presented by Shulevski et al. (2012; see Fig. 1) is here less pronounced. However, we can still recognize two enhancements in surface brightness along the eastern edge of the northern outer lobe and the western edge of the southern outer lobe close to the core.

In the SRT image at $6600 \mathrm{MHz}$, the northern outer lobe is clearly detected too, although not for its entire extension (see Fig. 2, bottom panel). Unfortunately, the low resolution of the map does not allow us to study the lobe sub-structures. The southern outer lobe is completely blended with the unrelated source in south-east direction as well with the central CSS source itself, preventing us from any further analysis.

\subsection{Spectral properties}

\subsubsection{Northern outer lobe}

To measure the spectral index of the northern lobe we have convolved the new LOFAR image and the WSRT image produced by Shulevski et al. (2012) to the resolution of the SRT map at $6600 \mathrm{MHz}$ equal to $2.9 \mathrm{arcmin}$. Because the instruments used in this work differ a lot from each other it is worth addressing the problem of the missing flux density. As this is not an issue for the single dish observations, it may become significant when using interferometers. The total flux density of an extended structure in a radio map can indeed be underestimated if the uv-plane does not provide enough uv-coverage at short spacings. An interferometer is sensitive to all the signal which is coming from structures in the sky with sizes $<0.6 \lambda / D_{\min }$, where $D_{\min }$ is its shortest baseline (Tamhane et al. 2015). The angular size of the northern lobe is $\sim 4.5 \operatorname{arcmin} \times 2.5 \mathrm{arcmin}$. The minimum observed baselines in the different observations are $D_{\min , 145 \mathrm{MHz}} \simeq 40 \mathrm{~m}$, $D_{\text {min, } 350 \mathrm{MHz}} \simeq 85 \mathrm{~m}$ and $D_{\min , 1400 \mathrm{MHz}} \simeq 30 \mathrm{~m}$ giving a largest angular scale $(\theta$, LAS $)$ equal to $\theta_{145 \mathrm{MHz}} \simeq 1.7 \mathrm{deg}, \theta_{350 \mathrm{MHz}} \simeq$ 21 arcmin and $\theta_{1400 \mathrm{MHz}} \simeq 14$ arcmin respectively. We, therefore, should be able to safely detect all the extended emission at all frequencies and making consistent comparisons between singledish and interferometric data.

We have extracted the flux density from one region drawn in correspondence of the northern lobe (region_north) as shown in Fig. 3 (top panel). The measured values are reported in Table 4. The errors on the flux densities are computed by combining in quadrature the flux scale error and the image noise as shown in Klein et al. (2003). The spectral index analysis in this region reveals a spectrum with very little curvature over the entire frequency range $145-6600 \mathrm{MHz}$ with $\alpha_{1400}^{145}=0.48 \pm 0.11$ and $\alpha_{6600}^{1400}=0.69 \pm 0.20$. The spectral curvature (SPC), defined as $\alpha_{6600}^{1400}-\alpha_{1400}^{145}$ (Murgia et al. 2011), is equal to $\mathrm{SPC} \simeq 0.2 \pm 0.2$, well below the typical value of SPC $>0.5$ expected for ageing plasma. Errors on the spectral indices are computed using the following expression:

$\alpha_{\mathrm{err}}=\frac{1}{\ln \frac{v_{1}}{v_{2}}} \sqrt{\left(\frac{S_{1, \mathrm{err}}}{S_{1}}\right)^{2}+\left(\frac{S_{2, \mathrm{err}}}{S_{2}}\right)^{2}}$

where $S_{1}$ and $S_{2}$ are the flux densities at frequencies $v_{1}$ and $v_{2}$ and $S_{1, \text { err }}$ and $S_{2, \text { err }}$ are the respective errors.
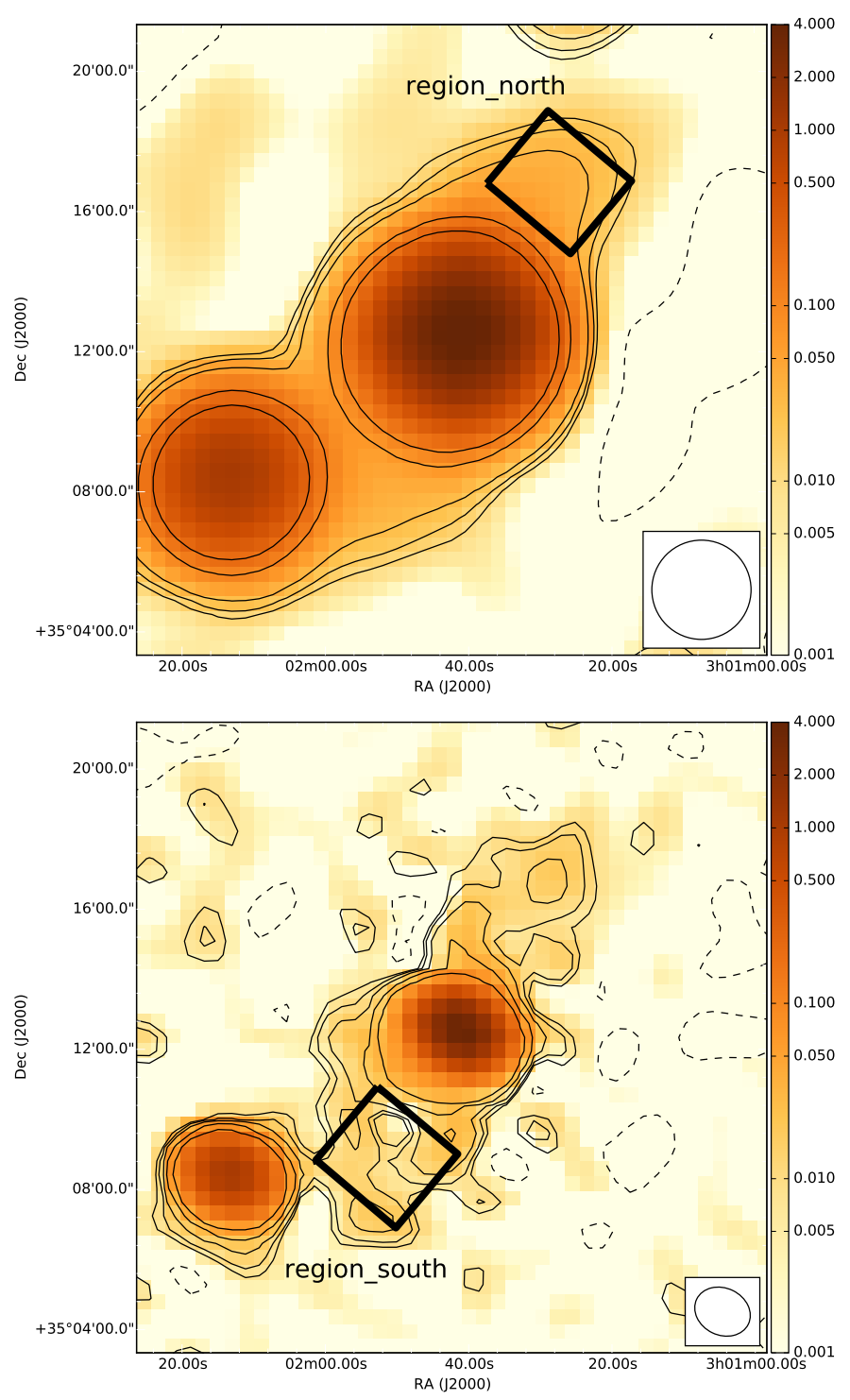

Fig. 3. Top panel: LOFAR image at $145 \mathrm{MHz}$ convolved to 2.9 -arcmin resolution. Contours represent $-2,2,3,5,10,20 \times \sigma\left(5 \mathrm{mJy} \mathrm{beam}^{-1}\right)$. Bottom panel: LOFAR image at $145 \mathrm{MHz}$ and $80 \operatorname{arcsec} \times 98$ arcsec resolution. Contours represent $-2,2,3,5,10,20 \times \sigma\left(3\right.$ mJy beam $\left.^{-1}\right)$. The black regions represent the boxes used to extract the flux density in the northern lobe and southern lobe respectively. The colour scale is set in Jy beam $^{-1}$.

\subsubsection{Southern outer lobe}

A measure of the flux density of the southern lobe is only possible at $145 \mathrm{MHz}$ and at $1400 \mathrm{MHz}$. To make this measurement we have convolved the WSRT image to the resolution of the LOFAR image of $80 \operatorname{arcsec} \times 98$ arcsec. We have extracted the flux density from one region drawn in correspondence of the southern lobe as shown in Fig. 3 (bottom panel, region_south). The measured values are reported in Table 4 . The spectral index is $\alpha_{1400}^{145}=0.73 \pm 0.07$ and is reported in Table 5 .

\subsection{Energetics and age of the outer radio lobes}

Using the observed properties of the outer lobe radio emission at different frequencies we can, to first order, evaluate some physical parameters of the radio plasma. 
Table 4. Flux densities of the northern lobe extracted from the radio images at 2.9-arcmin resolution and of the southern lobe extracted from the radio images at $80 \operatorname{arcsec} \times 98$ arcsec resolution.

\begin{tabular}{ccc}
\hline \hline Frequency & $\begin{array}{c}\text { Flux density } \\
\text { region_north } \\
(\mathrm{mJ})\end{array}$ & $\begin{array}{c}\text { Flux density } \\
\text { region_south } \\
(\mathrm{mJy})\end{array}$ \\
\hline 145 & $26.4 \pm 5.6$ & $68.1 \pm 8.0$ \\
1400 & $9.0 \pm 1.5$ & $13.4 \pm 1.3$ \\
6600 & $3.1 \pm 0.9$ & - \\
\hline
\end{tabular}

Notes. Region_north and region_south are shown in Fig. 3.

Table 5. Spectral indices computed for the northern lobe using region_north and for the southern lobe using region_south (see Table 4 and Fig. 3).

\begin{tabular}{ccc}
\hline \hline Spectral index & region_north & region_south \\
\hline$\alpha_{1400}^{145}$ & $0.48 \pm 0.11$ & $0.73 \pm 0.07$ \\
$\alpha_{6600}^{1400}$ & $0.69 \pm 0.20$ & - \\
$\alpha_{6600}^{145}$ & $0.56 \pm 0.09$ & - \\
\hline
\end{tabular}

Assuming equipartition conditions between particles and magnetic field, we have computed an average magnetic field value over the entire extension of the two outer lobes equal to $B_{\text {eq }}=1 \mu G$ (Worrall \& Birkinshaw 2006).

For this calculation we have assumed a power-law particle distribution of the form $N(\gamma) \propto \gamma^{-p}$ between a minimum and maximum Lorentz factor of $\gamma_{\min }=10$ and $\gamma_{\max }=10^{6}$, with $p$ being the particle energy power index. We have calculated the volume of the source assuming a cylindrical geometry in the plane of the sky. The radius and height of the cylinder were measured using the $3 \sigma$ contours as a reference in correspondence of the lobe maximum extension and are equal to $R=2.5 \mathrm{arcmin}$ and $h=12$ arcmin respectively. We have assumed that the particle energy content of the source to be equally distributed between heavy particles and electrons so that their ratio $k=1$, and we have set $p=2.0$ according to the observed low-frequency spectral shape where radiative energy losses are less significant. A value of $S_{1400}=120 \mathrm{mJy}$ is used following Shulevski et al. (2012). Moreover, this computation assumes that the magnetic field is uniformly distributed in the lobe volume.

By using the magnetic field $B_{\text {eq }}$ calculated in this way and a classical radiative ageing model (Kellermann 1964; Pacholczyk 1970) it is possible to get a first order estimate of the spectral age $t_{\mathrm{s}}$ of the particle population in the outer lobes. Indeed the age of the emitting particles remains encoded in the curvature of their radio spectrum. In particular, as the particles age with time, the spectrum gets progressively steeper at frequencies higher than a critical break frequency $v_{\mathrm{b}}$, due to preferential radiative cooling of high-energy particles. As already mentioned in Sect. 4.2, the spectrum of the northern lobe of the source B2 $0258+35$ only shows a little curvature over the entire observed frequency range 145-6600 MHz. This prevents us from properly modelling it to get a radiative age estimate. However, we can set a conservative upper limit to the source radiative age by assuming a lower limit to the spectral break equal to $1400 \mathrm{MHz}$, which corresponds to the highest frequency after which the spectrum starts showing a curvature. For this we use the following equation (Kardashev
1962; Murgia et al. 2011):

$t_{\mathrm{s}}=1590 \frac{B_{\mathrm{eq}}^{0.5}}{\left(B_{\mathrm{eq}}^{2}+B_{\mathrm{CMB}}^{2}\right) \sqrt{v_{\mathrm{b}}(1+z)}}$,

where $t_{\mathrm{s}}$ is in Myr, the magnetic field $B_{\text {eq }}$ and inverse Compton equivalent field $B_{\mathrm{CMB}}$ are in $\mu G$ and the break frequency $v_{\mathrm{b}}$ is in $\mathrm{GHz}$. We note that with such a low value of $B_{\text {eq }}$, the radiative cooling of the plasma is dominated by inverse Compton scattering of cosmic microwave background (CMB) photons. The $\mathrm{CMB}$ equivalent magnetic field equal to $B_{\mathrm{CMB}}=3.25 \times(1+z)^{2}$ has a value of $B_{\mathrm{CMB}}=3.36 \mu G$. In this way we get an upper limit on the lobe age equal to $\lesssim 110 \mathrm{Myr}$. The upper limit value of the radiative age is expected to decrease if the magnetic field was here underestimated due, for example, to an excess of non-radiative particles with respect to electrons. This occurrence is considered typical of low power jets, where entrainment of massive particles from the external IGM is dominant due to turbulence (Croston 2008; Massaglia et al. 2016). We also note that by using an alternative derivation of the magnetic field by Beck \& Krause (2005) the value lowers by almost a factor three, and this would cause the upper limit of the radiative age to increase. Finally, it should be kept in mind that some mechanisms, such as in situ particle re-acceleration, compression and mixing may affect this result as we discuss in Sect. 5.

\subsection{The central compact source}

The flux densities of the central compact source measured from our new images at different frequencies are listed in Table 6 and marked with an asterisk. The measurements have been performed on images having the same resolution at all frequencies (equal to 2.9 arcmin) by fitting a Gaussian function to the unresolved component. Flux densities at different frequencies collected from the literature using the NASA/IPAC Extragalactic Database are also listed in Table 6.

Thanks to the increased number of flux density measurements with respect to the work of Giroletti et al. (2005), especially at low frequency, we can now better recognize a spectral turnover between 100 and $200 \mathrm{MHz}$ and a possible spectral break at frequencies higher than $5000 \mathrm{MHz}$ (Fig. 4). We use the empirical relation between size and turnover frequency found by O'Dea \& Baum (1997) to measure the expected turnover frequency. This correlation has been for long explained in terms of synchrotron-self absorption related to the small size of the radio source (e.g. Snellen et al. 2000, Fanti 2009). We note, however, that free-free absorption due to a dense ambient medium is also thought to play a role according to some observations (e.g. Callingham et al. 2015; Tingay et al. 2015) and simulations (Bicknell et al. 2018). Under these circustances the following computed numbers must be treated with care.

Using the correlation presented in Orienti \& Dallacasa (2014) and a measured linear size for the CSS source equal to $3 \mathrm{kpc}$ we compute a predicted turnover frequency equal to $320 \mathrm{MHz}$. However, if we deproject the linear size of the source using the inclination angle of 45 deg estimated by Giroletti et al. (2005) we get a size of $4.3 \mathrm{kpc}$ and a corresponding turnover frequency equal to $260 \mathrm{MHz}$. Considered the large scatter present in the correlation (up to almost an order of magnitude), the result is consistent with the observations.

In order to better quantify the shape of the radio spectrum we have performed a spectral fitting using the software SYNAGE (Murgia et al. 1999). In particular, we have used the continuous injection spectral model $S_{\mathrm{CI}}(v)$ (Kardashev 1962) modified by 
Table 6. Flux densities of the central CSS source at different frequencies with respective references.

\begin{tabular}{lll}
\hline \hline $\begin{array}{l}\text { Frequency } \\
(\mathrm{MHz})\end{array}$ & $\begin{array}{l}\text { Flux } \\
(\mathrm{Jy})\end{array}$ & Reference \\
\hline 74 & $4.69 \pm 0.51$ & Cohen et al. (2007) \\
80 & $8.00 \pm 1.60$ & Slee (1995) \\
84 & $5.10 \pm 0.30$ & Hurley-Walker et al. (2017) \\
115 & $4.90 \pm 0.10$ & Hurley-Walker et al. (2017) \\
$145^{a}$ & $4.82 \pm 0.48$ & This work \\
150 & $4.50 \pm 0.10$ & Hurley-Walker et al. (2017) \\
151 & $5.11 \pm 0.17$ & Hales et al. (1993) \\
160 & $4.10 \pm 0.60$ & Slee (1995) \\
178 & $5.00 \pm 1.40$ & Pilkington \& Scott (1965) \\
$235^{a}$ & $4.06 \pm 0.20$ & This work \\
327 & $3.91 \pm 0.60$ & Rengelink et al. (1997) \\
$350^{a}$ & $3.51 \pm 0.18$ & This work \\
365 & $3.60 \pm 0.04$ & Douglas et al. (1996) \\
$612^{a}$ & $3.07 \pm 0.15$ & This work \\
1400 & $1.70 \pm 0.10$ & Brown et al. (2011) \\
1400 & $1.77 \pm 0.26$ & White \& Becker (1992) \\
1400 & $1.84 \pm 0.27$ & Condon et al. (1998) \\
1400 & $1.80 \pm 0.18$ & Shulevski et al. (2012) \\
1600 & $1.60 \pm 0.24$ & Sanghera et al. (1995) \\
2380 & $1.40 \pm 0.07$ & Dressel \& Condon (1978) \\
4835 & $0.90 \pm 0.10$ & Griffith et al. (1990) \\
4850 & $0.86 \pm 0.13$ & Gregory \& Condon (1991) \\
5000 & $0.92 \pm 0.14$ & Sanghera et al. (1995) \\
$6600^{a}$ & $0.73 \pm 0.07$ & This work \\
8400 & $0.61 \pm 0.05$ & Giroletti et al. (2005) \\
10700 & $0.58 \pm 0.03$ & Kellermann \& Pauliny-Toth (1973) \\
22500 & $0.250 \pm 0.005$ & Giroletti et al. (2005) \\
\hline & & \\
\hline
\end{tabular}

Notes. ${ }^{(a)}$ Observations presented in this work.

low-frequency synchrotron-self absorption (Pacholczyk 1970) presented in Murgia et al. (1999) and shown below:

$S(v) \propto\left(v / v_{1}\right)^{\alpha+\beta}\left(1-\mathrm{e}^{-\left(v / v_{1}\right)^{-(\alpha+\beta)}}\right) \cdot S_{C I}(v)$,

where $v_{1}$ is the frequency at which the optical depth is equal to 1 , $\alpha$ is the spectral index in the transparent frequency range and $\beta$ is the coefficient for an homogeneous synchrotron self-absorbed source and is fixed to 2.5 . For the fitting the flux density errors at all frequencies have been set to a systematic value equal to $10 \%$. We fix the injection index $\alpha_{\text {inj }}$ to a classical value of 0.5 and we find a break frequency equal to $v_{\mathrm{b}}=21800 \pm 8200 \mathrm{MHz}$ and a synchrotron self-absorption frequency equal to $v_{\mathrm{SSA}}=79 \pm$ $10 \mathrm{MHz}$. For comparison, we have also fitted the model setting the injection index $\alpha_{\text {inj }}$ as a free parameter. In this case we find a break frequency equal to $v_{\mathrm{b}}=6400 \pm 3700 \mathrm{MHz}$ and a synchrotron self-absorption frequency equal to $v_{\mathrm{SSA}}=65 \pm 24 \mathrm{MHz}$. We have used the statistical F-test to select the best model and find that the model with free $\alpha_{\text {inj }}$ does not improve the fitting with a confidence level of $95 \%$. Therefore, we consider the model with fixed $\alpha_{i n j}$ as the best fit model and we will refer to its results in the following discussion. The integrated spectrum of the central CSS source and the results from the best model fitting are presented in Fig. 4. Our new measurements, indicated as red squares in the figure, nicely follow the literature points, which are shown as black open circles.

The resulting $v_{\mathrm{b}}=21800 \pm 8200 \mathrm{MHz}$ is higher than the value estimated by Giroletti et al. (2005) equal to $4600 \mathrm{MHz}$. Assuming a magnetic field equal to $B_{\text {eq }}=90 \mu G$ (Giroletti et al. 2005) and using Eq. (2), we get an estimate of the particle radiative age

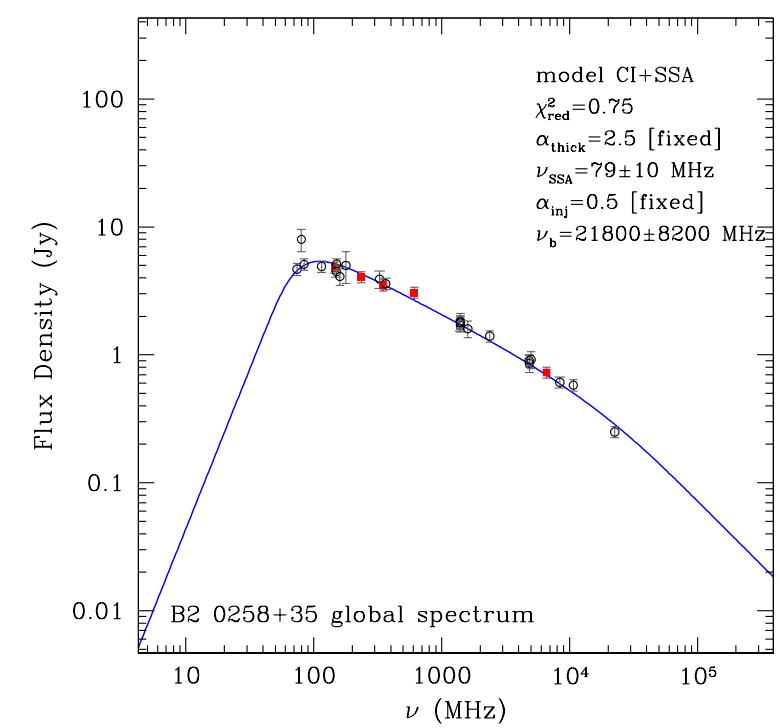

Fig. 4. Integrated radio spectrum of the central CSS source and best fit model. Red squares represent the flux density measurements presented in this paper, while black open circles are taken from literature. The entire list of flux densities used in the plot with respective errors and references is presented in Table 6 .

equal to 0.4 Myr. Despite the updated age for the CSS source is lower than the previous estimate by Giroletti et al. (2005) (0.9 Myr), it remains high when compared with sources of similar size studied in the literature (Murgia 2003). This may support a scenario where the expansion of the lobes is impeded by a dense interstellar medium as suggested by Giroletti et al. (2005) and further explored by the study of the HI gas by Murthy et al. (in prep.).

\section{Discussion}

Due to their very low surface brightness and amorphous morphology, the outer lobes of B2 $0258+35$ have been previously suggested to be old remnants of a past episode of AGN activity. The increase in frequency coverage presented in this work allows us to make a step forward in the understanding of the nature of these structures and in the overall radio galaxy evolution as we describe below.

\subsection{Source characteristics}

Our new LOFAR observations at $145 \mathrm{MHz}$ show that the outer lobes of B2 $0258+35$ have a total linear extension of $265 \mathrm{kpc}$ and a relaxed shape with low surface brightness $\left(4.7 \mathrm{mJy} \mathrm{arcmin}^{-2}\right.$ at $\left.145 \mathrm{MHz}\right)$ in agreement with the previous study at $1400 \mathrm{MHz}$ (Shulevski et al. 2012). The surface brightness values are consistent with other observations of remnant radio lobes (e.g. $4 \mathrm{mJy} \operatorname{arcmin}^{-2}$, Brienza et al. 2016; 2.5-6 mJy $\operatorname{arcmin}^{-2}$, Saripalli et al. 2012), a few giant radio galaxy lobes (e.g. $1 \mathrm{mJy} \operatorname{arcmin}^{-2}$, Saripalli et al. 2012; $10 \mathrm{mJy} \mathrm{arcmin}^{-2}$, Subrahmanyan et al. 2006) and the radio galaxy NGC 3998 (5 mJy arcmin ${ }^{-2}$, Frank et al. 2016). The outer lobes are so faint that they only represent $\sim 6 \%$ of the entire source luminosity. This extreme contrast in surface brightness between the inner and outer structure represents, to date, a quite rare case among the radio galaxy population as further discussed is Sect. 5.3. Because the host galaxy is located in a low density environment, the plasma of the outer lobes may have suffered a 
severe adiabatic expansion and a consequent surface brightness decrease. However, if this is the case, we would expect the outer lobes to dissipate very quickly in the ambient medium. Highsensitivity X-ray observations would be needed to probe the pressure balance between the lobes and the surrounding IGM. At the same time, if the inner jets have strong interactions with the surrounding ISM, the radio luminosity may be boosted due to compression of the magnetic field and the increased particle density (Morganti et al. 2011; Tadhunter et al. 2011; Giroletti et al. 2005).

Other features that are interesting to discuss and may provide hints for the interpretation of the source are the broad enhancements in surface brightness present at the edges of the outer lobes. One possible explanation is that this S-shape structure represents an active channel of plasma connecting the inner and outer lobes. Alternatively, it could represent remnant large-scale jets from the previous cycle of activity. The reason for the bent geometry of these features remains unclear though. One possibility is that the jets are bent as a result of the strong interaction with the dense nuclear ISM. Otherwise the jets may have been subject to precession. Precession of radio jets have been observed in radio galaxies (e.g. Falceta-Gonçalves et al. 2010; Gong et al. 2011; Nawaz et al. 2016). Models show that on such large spatial scales precession can only happen on timescales larger than 1-10 Myr (e.g. Nixon \& King 2013), which would be compatible with the timescales we have estimated for the radio galaxy B2 $0258+35$. The origin of the phenomenon is typically connected to gas misalignments in the accretion disk, which are often a consequence of a merger event, although it is still not completely clear how the timescales of these instabilities and the precession of kiloparsec-scale jets relate (e.g. Lu \& Zhou 2005). Whether the source B2 $0258+35$ has actually experienced accretion instabilities is difficult to assess. Shulevski et al. (2012) investigate extensively different scenarios for the black hole gas supply and triggering based on the results of the gas kinematics of Struve et al. (2010), Emonts (2006) and Prandoni et al. (2007). From that, they exclude a direct link between the merger history of the source and the duty cycle of the radio activity. They suggest instead that the black hole is accreting gas that is cooling from the galaxy's hot corona via Bondi accretion (Bondi 1952; Hardcastle et al. 2007). In this case, chaotic accretion of cold gas clouds condensed from the hot atmosphere may also take place as suggested by a few authors (e.g. Soker et al. 2009; Pizzolato \& Soker 2010; Gaspari et al. 2013, 2017). This may be responsible for the accretion instabilities required by precession models, as well as they could justify the jet time discontinuity. Moreover, we cannot exclude that some interactions with the small gas-rich satellites that surround the host galaxy may have disturbed the accretion process and influenced the AGN duty cycle.

Beside the morphology, another interesting property of the outer lobes is the spectral shape. We find that the radio spectrum of neither the northern lobe nor the southern lobe is ultrasteep $(\alpha>1.2)$, as expected for an old ageing plasma. Moreover, despite the wide frequency coverage available for the northern lobe $(145-6600 \mathrm{MHz})$, no significant spectral curvature is identified (see Sect. 4.2). Based on the spectral slope and using a simple radiative model, we have derived a first order upper limit to the plasma age in the northern lobe equal to $<110 \mathrm{Myr}$ (see Sect. 4.3). We stress that if mechanisms such as particle re-acceleration, mixing or compression are in action, this age estimate does not hold anymore as it is further discussed in Sect. 5.2). By treating the lobes as buoyant bubbles expanding in the intergalactic medium (IGM) with a rising speed of $\sim 613 \mathrm{~km} \mathrm{~s}^{-1}$, Shulevski et al. (2012) have estimated that the time elapsed since the jet activity has ceased is equal to $\gtrsim 80 \mathrm{Myr}$ (the lower limit is introduced to take into account a possible inclination angle). This value, however, can also be interpreted as an upper limit to the entire age of the outer lobes if we consider that the outer lobes must have expanded to the current size already during the jet activity. In this case the lobes, pushed by the jets advance, should have expanded faster than what assumed by the buoyancy scenario. With this assumption the dynamical age of the outer lobes is consistent with the radiative age upper limit computed in this work.

\subsection{Source evolutionary scenarios and duty cycle}

The characteristics of the source B2 $0258+35$ are exceptional and a unique interpretation of the source's evolution remains difficult to assess. Here we propose three evolutionary scenarios to explain the spectral and morphological properties discussed above.

1. The radio AGN has never switched off. Instead, the largescale jets have been temporarily disrupted and smothered by e.g. the interaction of the particle flows with a dense medium as suggested by Baum et al. (1990). In this case the two enhancements in surface brightness visible at the edges of the outer lobes could represent two uncollimated jets that connect the outer and inner structure. Under this circumstance the outer lobes would still be fuelled with fresh particles, even if at a low rate, and this would explain the small spectral curvature. This scenario may be consistent with the idea of the inner lobes currently being confined or impeded by a dense interstellar medium as suggested by Giroletti et al. (2005) and Murty et al. (in prep.).

2. The radio AGN has actually stopped and restarted after a short amount of time. The outer lobes could be currently disconnected from the inner jets and therefore not replenished with fresh particles. However, if the time elapsed since the AGN switch off is not too long, it is possible that the spectral steepening produced by particle ageing in the outer lobes is still not prominent in the considered spectral range (145-6600 MHz). The low level of magnetic field observed would also contribute to make the spectral evolution slow. In this case the two enhancements in surface brightness discussed above could be interpreted as the channels of the previously active jets that have recently stopped to be fuelled. Assuming this scenario, we can use the timescales presented in Sect. 4.3 to place an upper limit to the time elapsed between the two phases of jet activity equal to $\lesssim 100 \mathrm{Myr}$, by combining the radiative age estimate for the outer lobes $(<110 \mathrm{Myr}$, see Sect. 4.3) with the CSS radiative age equal to $0.4 \mathrm{Myr}$ (see Sect. 4.4). This is in agreement with the initial estimate by Shulevski et al. (2012). Higher frequency observations would be needed to set tighter constraints on the radiative age of the outer lobes and therefore on the duty cycle. Unfortunately such high sensitivity observations on such large spatial scales are difficult to obtain.

3. The outer lobes are remnants of a previous AGN activity and are detached from the current nuclear activity but they do not show an important spectral curvature because of mechanisms such as in situ particle reacceleration, adiabatic compression and particle mixing. All these mechanisms represent valid alternatives to explain the small spectral curvature as they influence the spectral evolution. We stress that, in this case, the computation of the radiative age presented above is not valid. In particular, particle re-acceleration tend to 
increase the energy of high energy particles causing a "flattening" of the high-frequency tail of the observed radio spectrum (Alexander 1987). This phenomenon may be caused by shocks, like backflows in lobes of active radio galaxies, or stochastic processes, like plasma turbulence. In this occurrences we would also expect significant mixing of particles of different ages within the outer lobes and this would contribute to erase the spectral curvature (Turner et al. 2018). By increasing the kinetic energy of the particle population and thus shifting the break frequency to higher frequencies, adiabatic compression may also have a role in contaminating the radiative age estimate (Scheuer \& Williams 1968; Alexander 1987). In this case, Alexander (1987) suggests that some brightness enhancement should be observed together with some polarized emission produced by the compressed magnetic plasma (Laing 1980).

For the source B2 $0258+35$, re-acceleration and particle mixing may be compatible with the presence of turbulence created as a consequence of the shock evolution, between the low-power jets and the ambient medium (Croston 2008; Massaglia et al. 2016). Adiabatic compression also represents an interesting possibility. As mentioned in Sect. 4.1, the outer lobes show at both 145 and $1400 \mathrm{MHz}$ two surface brightness enhancements at the edge of each lobe in specular position with respect to the core that could fit the expected features for adiabatic compression. Polarization information will be used to test this hypothesis (Adebahr et al., in prep.). Discriminating among the above-mentioned scenarios and providing tight constrain on the duty cycle of B2 $0258+35$ is difficult. However, our results seem to suggest that in no case the outer lobes are compatible with very old remnants as the morphology alone would suggest and as it was previously proposed. Indeed, all of the mechanisms considered above to explain the small spectral curvature become irrelevant after some time after the large scale jets have switched off. For example Eilek (2014) estimates that, for the case of Centaurus A, the decay time of the turbulence is of the order of few tens of Myr after which the lobes should quickly fade away. By assuming comparable values of turbulence decay for B2 $0258+35$, it follows that, either the lobes are still fuelled by the nuclear activity or the switch off must have happened only few tens of Myr ago, suggesting a quiescent phase shorter than a few tens of Myr, contrary to previous expectations.

\subsection{Comparison with other radio galaxies}

As already mentioned above, the properties of B2 $0258+35$ are peculiar among radio galaxies. However, we have recognized a small number of objects that show some comparable characteristics as described below.

A few sources, for example, show some inner and outer lobes with a very high surface brightness contrast as observed in B2 $0258+35$ and for this reason are claimed to be restarted radio galaxies. Among these there are the radio galaxy associated with NGC 3998 ( $20 \mathrm{kpc}$, Frank et al. 2016), the source 4C 29.30 ( 600 kpc, Jamrozy et al. 2007), the misaligned DDRG 3C293 (190 kpc, Joshi et al. 2011; Machalski et al. 2016), the source Mrk 6 (7.5 kpc, Kharb et al. 2006) and the well-known radio galaxy Centaurus A $(\sim 200 \mathrm{kpc}$, McKinley et al. 2018; Morganti et al. 1999). Moreover, we note some similarity with the famous radio galaxies M87 (e.g. Owen et al. 2000) and 3C317 (Zhao et al. 1993; Venturi et al. 2004). Particularly interesting is the halo of the source M87, which seems to be made of a superposition of many plasma bubbles of different ages and does not show any significant spectral curvature in its spectrum, with no clear relation between the spectral index and the brightness distribution at low frequencies (de Gasperin et al. 2012). It is interesting to note that some of these sources, that is, NGC 3998, Mrk 6 and Centaurus A show a similar S-shape morphology to B2 $0258+35$. The origin of this shape has been suggested to be related to a change of angular momentum in the accreting gas or to an episodically powered precessing jet (Frank et al. 2016; Kharb et al. 2006).

From a spectral point of view we find a striking resemblance between B2 $0258+35$ and Centaurus A. Indeed, the radio spectrum of the northern outer lobe of Centaurus A is not ultra-steep up to $90000 \mathrm{MHz}$ (Hardcastle et al. 2009; Alvarez et al. 2000) and its age is estimated to range between few tens of Myr (using radiative models, Hardcastle et al. 2009) and $>100 \mathrm{Myr}$ (using dynamical models, Saxton et al. 2001). While, in situ particle reacceleration is claimed to be a key component for preventing the spectrum from steepening at high frequency, the discovery of an intermediate-scale northern middle lobe (Morganti et al. 1999; McKinley et al. 2013, 2018) suggests the presence of an "open channel" of fresh particles between the inner and the outer lobe.

While all the afore-mentioned radio galaxies show signs of recurrent jet activity, the origin and timescales of this intermittence are still unclear. The sources 3C317 and M87 are located at the centre of massive clusters of galaxies so they are most likely going through a short, self-regulating AGN feedback cycle with the surrounding hot medium.

All the other sources presented above, including B2 $0258+35$, are located instead in low density environments. Therefore, their jet activity is expected to be triggered less frequently. It is worth noting though, that they all show signatures of past merger events and a large reservoir of gas in different phases (e.g. HI, CO).

Mergers are often claimed to be responsible for the triggering of the AGN in galaxies. However, the timescales of the radio activity are usually found to be much shorter $\left(10^{6}-10^{7} \mathrm{yr}\right)$ than those computed for the merger events $\left(10^{8}-10^{9} \mathrm{yr}\right)$, making the connection between the two phenomena weak (e.g. Emonts 2006; Struve et al. 2010; Maccagni et al. 2014). More compatible timescales $\left(10^{5}-10^{7} \mathrm{yr}\right)$ come from multiple galaxy encounters that preceed the merger event and can increase the gas kinematical instabilities in the host galaxy, as suggested for explaining the origin of DDRGs (Schoenmakers et al. 2000).

Alternatives that do not require any external contribution are instabilities in the accretion disk, for instance caused by the radiation pressure induced warping (Pringle 1997; Czerny et al. 2009), or chaotic accretion of cold gas clouds (Soker et al. 2009; Gaspari et al. 2013, 2017). This last scenario looks particularly promising especially in gas rich galaxies like those analysed here. Indeed, local instabilities occurring in the observed gas structures (such as the HI disk in B2 $0258+35$ ) may cause an infall of clouds towards the galaxy centre, which may trigger the AGN frequently and intermittently (see Soker et al. 2009; Gaspari et al. 2013, 2017).

\section{Conclusions and future work}

In this paper we have presented new multi-frequency observations of the source B2 $0258+35$ aimed at investigating the properties of the outer lobes initially identified by Shulevski et al. (2012) at $1400 \mathrm{MHz}$. The lobes are further detected at $145 \mathrm{MHz}$ with LOFAR and at $6600 \mathrm{MHz}$ with SRT. The main findings are summarized below: 
(i) New observations with LOFAR at $145 \mathrm{MHz}$ confirm the size $(\sim 265 \mathrm{kpc})$, relaxed shape and low surface brightness of the outer lobes previously observed at $1400 \mathrm{MHz}$ by Shulevski et al. (2012). The emission from the outer lobes only represent $\sim 6 \%$ of the entire source luminosity at $145 \mathrm{MHz}$. The extreme luminosity contrast between the inner and outer structure might be explained, on one hand, by strong adiabatic expansion of the plasma of the outer lobes in the ambient medium and, on the other hand, by a luminosity boost of the inner lobes due to compression of the magnetic field and the increased density of particles.

(ii) The S-shape morphology observed in the map at $1400 \mathrm{MHz}$ is visible in the LOFAR map too, although much less pronounced due to the lower angular resolution. This structure may be interpreted as due to low power jets that connect the inner and outer lobes or to jet remnants from the previous cycle of activity. The S-shape may have originated from the jets bending due to a dense ISM or from precession.

(iii) The combination of the new LOFAR and SRT observations with the data at $1400 \mathrm{MHz}$ shows that the integrated spectrum of the outer northern lobe is not ultra steep and does not present a significant spectral curvature (SPC $\sim 0.2 \pm 0.2$ ) with spectral indices equal to $\alpha_{1400}^{145}=0.48 \pm 0.11$ and $\alpha_{6600}^{1400}=0.69 \pm$ 0.20 . The spectral index in the outer southern lobe in the range $145-1400 \mathrm{MHz}$ is $\alpha_{1400}^{145}=0.73 \pm 0.07$.

(iv) By assuming a simple radiative model to describe the integrated spectrum of the outer northern lobe we have computed a first order upper limit to the radiative age equal to $\lesssim 110 \mathrm{Myr}$. This value is compatible with the dynamical age computed by Shulevski et al. (2012) and provides an estimate of the jet quiescent time between the two subsequent phases of activity equal to $\lesssim 100$ Myr. We stress that this value should be treated with care as it neglects mechanisms such as situ particle reacceleration, mixing and/or adiabatic compression that may alter the spectral shape.

(v) Possible evolutionary scenarios to explain the source morphology and spectral properties of the outer lobes are the following: (1) the AGN has never switched off but the large-scale radio jets have only been temporarily disrupted. The outer lobes are still fuelled by fresh particles from the nucleus at a very low rate; (2) the active nucleus has switched off and on again after a short time so that the plasma in the outer lobes is still not aged; (3) the outer lobes are remnants of a previous AGN activity but in situ particle reacceleration, mixing and/or adiabatic compression prevent their spectrum from further steepening.

(vi) As all the mechanisms mentioned above stop to be relevant on timescales longer than a few tens of Myr, it follows that the outer lobes are not very old remnants as the morphology alone would suggest and as it was previously claimed. Instead, we propose that either the outer lobes are still fuelled by the nuclear activity or the switch off must have happened only few tens of Myr ago, suggesting a jet quiescent phase shorter than a few tens of Myr.

(vii) Future searches of restarted radio galaxies should bear in mind that not all low-surface brightness lobes and halos with amorphous shape that are found around compact, bright jets are necessarily ultra-steep spectrum remnants of past AGN activity. This is especially true for low power radio galaxies.

The morphological and the spectral characteristics of the source B2 $0258+35$ are quite exceptional and only a small number of other radio galaxies show some resemblance. Analysis of its polarization properties will be used to further investigate the nature of the outer lobes and the source evolution (Adebahr et al. in prep.).
To date, it is not clear whether these sources are intrinsically rare or whether they have just been missed by the old-generation instruments, due to their lack of sensitivity, especially at low frequency. LOFAR is the perfect instrument to clarify this as it provides at the same time high spatial resolution $(20$ arcsec up to 6 arcsec) and high sensitivity (with typical noise values up to $0.1 \mathrm{mJy}$ beam $^{-1}$ in $8 \mathrm{~h}$ observations) at $145 \mathrm{MHz}$. By using LoTSS we are starting a systematic search for low surface brightness, extended emission around a sample of well-known compact radio galaxies $(<$ few $\mathrm{kpc}$ ) that are located in the survey area. Low frequency data will be able to probe even the most aged particle population. With this we will get a better statistics of the presence of such extended structures around compact radio galaxies and we will be able to investigate their properties and nature in a statistical way.

Acknowledgements. The research leading to these results has received funding from the European Research Council under the European Union's Seventh Framework Programme (FP/2007-2013) / ERC Advanced Grant RADIOLIFE320745. LOFAR, the Low Frequency Array designed and constructed by ASTRON (Netherlands Institute for Radio Astronomy), has facilities in several countries, that are owned by various parties (each with their own funding sources), and that are collectively operated by the International LOFAR Telescope (ILT) foundation under a joint scientific policy. The Sardinia Radio Telescope is funded by the Department of University and Research (MIUR), Italian Space Agency (ASI), and the Autonomous Region of Sardinia (RAS) and is operated as National Facility by the National Institute for Astrophysics (INAF). The development of the SARDARA back-end has been funded by the Autonomous Region of Sardinia (RAS) using resources from the Regional Law 7/2007 "Promotion of the scientific research and technological innovation in Sardinia" in the context of the research project CRP-49231 (year 2011, PI: Possenti): "High resolution sampling of the Universe in the radio band: an unprecedented instrument to understand the fundamental laws of the nature". We thank the staff of the GMRT that made these observations possible. GMRT is run by the National Centre for Radio Astrophysics of the Tata Institute of Fundamental Research. The National Radio Astronomy Observatory is a facility of the National Science Foundation operated under cooperative agreement by Associated Universities, Inc. This research has made use of the NASA/IPAC Extragalactic Database (NED), which is operated by the Jet Propulsion Laboratory, California Institute of Technology, under contract with the National Aeronautics and Space Administration. This research made use of APLpy, an open-source plotting package for Python hosted at http: //aplpy.gi thub.com.

\section{References}

Alexander, P. 1987, MNRAS, 225, 27

Alvarez, H., Aparici, J., May, J., \& Reich, P. 2000, A\&A, 355, 863 Baum, S. A., O'Dea, C. P., Murphy, D. W., \& de Bruyn, A. G. 1990, A\&A, 232, 19

Beck, R., \& Krause, M. 2005, Astron. Nachr., 326, 414

Best, P. N., Kauffmann, G., Heckman, T. M., et al. 2005, MNRAS, 362, 25

Bhatnagar, S., Cornwell, T. J., Golap, K., \& Uson, J. M. 2008, A\&A, 487, 419

Bicknell, G. V., Mukherjee, D., Wagner, A. Y., Sutherland, R. S., \& Nesvadba, N. P. H. 2018, MNRAS, 475, 3493

Bolli, P., Orlati, A., Stringhetti, L., et al. 2015, J. Astron. Instrum., 4, 1550008 Bondi, H. 1952, MNRAS, 112, 195

Brienza, M., Godfrey, L., Morganti, R., et al. 2016, A\&A, 585, A29 Brienza, M., Godfrey, L., Morganti, R., et al. 2017, A\&A, 606, A98 Brown, M. J. I., Jannuzi, B. T., Floyd, D. J. E., \& Mould, J. R. 2011, ApJ, 731, L41

Callingham, J. R., Gaensler, B. M., Ekers, R. D., et al. 2015, ApJ, 809, 168

Cohen, A. S., Lane, W. M., Cotton, W. D., et al. 2007, AJ, 134, 1245

Condon, J. J., Cotton, W. D., Greisen, E. W., et al. 1998, AJ, 115, 1693

Cornwell, T. J., \& Perley, R. A. 1992, A\&A, 261, 353

Croston, J. H. 2008, in Extragalactic Jets: Theory and Observation from Radio to Gamma Ray, eds. T. A. Rector, \& D. S. De Young, ASP Conf. Ser., 386, 335

Czerny, B., Siemiginowska, A., Janiuk, A., Nikiel-Wroczyński, B., \& Stawarz, Ł. 2009, ApJ, 698, 840

de Gasperin, F., Orrú, E., Murgia, M., et al. 2012, A\&A, 547, A56

Di Matteo, T., Springel, V., \& Hernquist, L. 2005, Nature, 433, 604

Douglas, J. N., Bash, F. N., Bozyan, F. A., Torrence, G. W., \& Wolfe, C. 1996, AJ, 111, 1945 
Dressel, L. L., \& Condon, J. J. 1978, ApJS, 36, 53

Eilek, J. A. 2014, New J. Phys., 16, 045001

Emonts, B. H., 2006, PhD Thesis, University of Groningen, The Netherlands

Emonts, B. H. C., Morganti, R., Struve, C., et al. 2010, MNRAS, 406, 987

Fabian, A. C. 2012, ARA\&A, 50, 455

Falceta-Gonçalves, D., Caproni, A., Abraham, Z., Teixeira, D. M., \& de Gouveia Dal Pino, E. M. 2010, ApJ, 713, L74

Fanti, C. 2009, Astron. Nachr., 330, 120

Frank, B. S., Morganti, R., Oosterloo, T., Nyland, K., \& Serra, P. 2016, A\&A, 592, A94

Gaspari, M., Brighenti, F., \& Temi, P. 2012, MNRAS, 424, 190

Gaspari, M., Ruszkowski, M., \& Oh, S. P. 2013, MNRAS, 432, 3401

Gaspari, M., Temi, P., \& Brighenti, F. 2017, MNRAS, 466, 677

Giovannini, G., Cotton, W. D., Feretti, L., Lara, L., \& Venturi, T. 2001, ApJ, 552, 508

Giroletti, M., Giovannini, G., \& Taylor, G. B. 2005, A\&A, 441, 89

Godfrey, L. E. H., Morganti, R., \& Brienza, M. 2017, MNRAS, 471, 891

Gong, B. P., Li, Y. P., \& Zhang, H. C. 2011, ApJ, 734, L32

Govoni, F., Murgia, M., Vacca, V., et al. 2017, A\&A, 603, A122

Gregory, P. C., \& Condon, J. J. 1991, ApJS, 75, 1011

Griffith, M., Langston, G., Heflin, M., et al. 1990, ApJS, 74, 129

Hales, S. E. G., Baldwin, J. E., \& Warner, P. J. 1993, MNRAS, 263, 25

Hardcastle, M. J., Evans, D. A., \& Croston, J. H. 2007, MNRAS, 376, 1849

Hardcastle, M. J., Cheung, C. C., Feain, I. J., \& Stawarz, Ł. 2009, MNRAS, 393, 1041

Heald, G., McKean, J., Pizzo, R., et al. 2010, in ISKAF2010 Science Meeting, 57

Ho, L. C., Filippenko, A. V., \& Sargent, W. L. W. 1997, ApJS, 112, 315

Hurley-Walker, N., Johnston-Hollitt, M., Ekers, R., et al. 2015, MNRAS, 447, 2468

Hurley-Walker, N., Callingham, J. R., Hancock, P. J., et al. 2017, MNRAS, 464, 1146

Intema, H. T. 2014, Astron. Soc. India Conf. Ser., 13

Intema, H. T., Jagannathan, P., Mooley, K. P., \& Frail, D. A. 2017, A\&A, 598, A78

Jamrozy, M., Konar, C., Saikia, D. J., et al. 2007, MNRAS, 378, 581

Jamrozy, M., Saikia, D. J., \& Konar, C. 2009, MNRAS, 399, L141

Joshi, S. A., Nandi, S., Saikia, D. J., Ishwara-Chandra, C. H., \& Konar, C. 2011, MNRAS, 414, 1397

Kaiser, C. R., Schoenmakers, A. P., \& Röttgering, H. J. A. 2000, MNRAS, 315 , 381

Kardashev, N. S. 1962, Sov. Astron., 6, 317

Kellermann, K. I. 1964, ApJ, 140, 969

Kellermann, K. I., \& Pauliny-Toth, I. I. K. 1973, AJ, 78, 828

Kharb, P., O’Dea, C. P., Baum, S. A., Colbert, E. J. M., \& Xu, C. 2006, ApJ, 652 , 177

Klein, U., Mack, K.-H., Gregorini, L., \& Vigotti, M. 2003, A\&A, 406, 579

Konar, C., \& Hardcastle, M. J. 2013, MNRAS, 436, 1595

Konar, C., Hardcastle, M. J., Jamrozy, M., \& Croston, J. H. 2013, MNRAS, 430, 2137

Kuźmicz, A., Jamrozy, M., Kozieł-Wierzbowska, D., \& Weżgowiec, M. 2017, MNRAS, 471, 3806

Laing, R. A. 1980, MNRAS, 193, 439

Loi, F., Murgia, M., Govoni, F., et al. 2017, MNRAS, 472, 3605

Lu, J.-F., \& Zhou, B.-Y. 2005, ApJ, 635, L17

Luo, W. F., Yang, J., Cui, L., Liu, X., \& Shen, Z. Q. 2007, Chin. J. Astron. Astrophys., 7, 611

Maccagni, F. M., Morganti, R., Oosterloo, T. A., \& Mahony, E. K. 2014, A\&A, 571, A67

Machalski, J., Jamrozy, M., Stawarz, Ł., \& Weżgowiec, M. 2016, A\&A, 595, A46

Mahatma, V. H., Hardcastle, M. J., Williams, W. L., et al. 2018, MNRAS, 475, 4557

Mahony, E. K., Morganti, R., Prandoni, I., et al. 2016, MNRAS, 463, 2997

Massaglia, S., Bodo, G., Rossi, P., Capetti, S., \& Mignone, A. 2016, A\&A, 596, A12

McKinley, B., Briggs, F., Gaensler, B. M., et al. 2013, MNRAS, 436, 1286

McKinley, B., Tingay, S. J., Carretti, E., et al. 2018, MNRAS, 474, 4056

McMullin, J. P., Waters, B., Schiebel, D., Young, W., \& Golap, K. 2007, in Astronomical Data Analysis Software and Systems XVI, eds. R. A. Shaw, F. Hill, \& D. J. Bell, ASP Conf. Ser., 376, 127

McNamara, B. R., \& Nulsen, P. E. J. 2007, ARA\&A, 45, 117

Melis, A., Concu, R., Trois, A., et al. 2018, J. Astron. Instrum., 7, 1850004

Morganti, R. 2017, Nat. Astron., 1, 596
Morganti, R., Killeen, N. E. B., Ekers, R. D., \& Oosterloo, T. A. 1999, MNRAS, 307,750

Morganti, R., Holt, J., Tadhunter, C., et al. 2011, A\&A, 535, A97

Murgia, M. 2003, PASA, 20, 19

Murgia, M., Fanti, C., Fanti, R., et al. 1999, A\&A, 345, 769

Murgia, M., Parma, P., Mack, K.-H., et al. 2011, A\&A, 526, A148

Murgia, M., Govoni, F., Carretti, E., et al. 2016, MNRAS, 461, 3516

Nawaz, M. A., Bicknell, G. V., Wagner, A. Y., Sutherland, R. S., \& McNamara, B. R. 2016, MNRAS, 458, 802

Nixon, C., \& King, A. 2013, ApJ, 765, L7

Noordermeer, E., van der Hulst, J. M., Sancisi, R., Swaters, R. A., \& van Albada, T. S. 2005, A\&A, 442, 137

O'Dea, C. P., \& Baum, S. A. 1997, AJ, 113, 148

Orienti, M., \& Dallacasa, D. 2008, A\&A, 487, 885

Orienti, M., \& Dallacasa, D. 2014, MNRAS, 438, 463

Orrù, E., van Velzen, S., Pizzo, R. F., et al. 2015, A\&A, 584, A112

Owen, F. N., Eilek, J. A., \& Kassim, N. E. 2000, ApJ, 543, 611

Pacholczyk, A. G. 1970, Radio Astrophysics. Nonthermal Processes in Galactic and Extragalactic Sources (San Francisco: Freeman)

Parma, P., Murgia, M., de Ruiter, H. R., et al. 2007, A\&A, 470, 875

Perley, R. A., \& Butler, B. J. 2013, ApJS, 204, 19

Pilkington, J. D. H., \& Scott, J. F. 1965, MNRAS, 69, 183

Pizzolato, F., \& Soker, N. 2010, MNRAS, 408, 961

Prandoni,, I., Laing, R. A.,, Parma, P., et al. 2007, in From Z-Machines to ALMA: (Sub)Millimeter Spectroscopy of Galaxies, eds. A. J. Baker, J. Glenn, A. I. Harris, J. G. Mangum, \& M. S. Yun, ASP Conf. Ser., 375, 271

Prandoni, I., Murgia, M., Tarchi, A., et al. 2017, A\&A, 608, A40

Pringle, J. E. 1997, MNRAS, 292, 136

Rengelink, R. B., Tang, Y., de Bruyn, A. G., et al. 1997, A\&AS, 124, 259

Saikia, D. J., \& Jamrozy, M. 2009, Bull. Astron. Soc. India, 37, 63

Sanghera, H. S., Saikia, D. J., Luedke, E., et al. 1995, A\&A, 295, 629

Saripalli, L., Subrahmanyan, R., Thorat, K., et al. 2012, ApJS, 199, 27

Saxton, C. J., Sutherland, R. S., \& Bicknell, G. V. 2001, ApJ, 563, 103

Scaife, A. M. M., \& Heald, G. H. 2012, MNRAS, 423, L30

Schaye, J., Crain, R. A., Bower, R. G., et al. 2015, MNRAS, 446, 521

Scheuer, P. A. G., \& Williams, P. J. S. 1968, ARA\&A, 6, 321

Schoenmakers, A. P., de Bruyn, A. G., Röttgering, H. J. A., van der Laan, H., \& Kaiser, C. R. 2000, MNRAS, 315, 371

Shabala, S. S., Ash, S., Alexander, P., \& Riley, J. M. 2008, MNRAS, 388, 625

Shimwell, T. W., Röttgering, H. J. A., Best, P. N., et al. 2017, A\&A, 598, A104

Shulevski, A., Morganti, R., Oosterloo, T., \& Struve, C. 2012, A\&A, 545, A91

Shulevski, A., Morganti, R., Harwood, J. J., et al. 2017, A\&A, 600, A65

Sijacki, D., Vogelsberger, M., Genel, S., et al. 2015, MNRAS, 452, 575

Slee, O. B. 1995, Aust. J. Phys., 48, 143

Snellen, I. A. G., Schilizzi, R. T., Miley, G. K., et al. 2000, MNRAS, 319, 445

Soker, N., Sternberg, A., \& Pizzolato, F. 2009, AIP Conf. Ser., 1201, 321

Struve, C., Oosterloo, T. A., Morganti, R., \& Saripalli, L. 2010, A\&A, 515, A67

Subrahmanyan, R., Hunstead, R. W., Cox, N. L. J., \& McIntyre, V. 2006, ApJ, 636, 172

Swarup, G. 1991, in IAU Colloq. 131: Radio Interferometry. Theory, Techniques, and Applications, eds. T. J. Cornwell, \& R. A. Perley, ASP Conf. Ser., 19, 376

Tadhunter, C., Holt, J., González Delgado, R., et al. 2011, MNRAS, 412, 960

Tamhane, P., Wadadekar, Y., Basu, A., et al. 2015, MNRAS, 453, 2438

Tasse, C., van der Tol, S., van Zwieten, J., van Diepen, G., \& Bhatnagar, S. 2013, A\&A, 553, A105

Tingay, S. J., Macquart, J.-P., Collier, J. D., et al. 2015, AJ, 149, 74

Turner, R. J., \& Shabala, S. S. 2015, ApJ, 806, 59

Turner, R. J., Rogers, J. G., Shabala, S. S., \& Krause, M. G. H. 2018, MNRAS, 473, 4179

Vacca, V., Murgia, M., \& Loi, F. G. F. 2018, MNRAS, 479, 776

van Haarlem, M. P., Wise, M. W., Gunst, A. W., et al. 2013, A\&A, 556, A2

van Weeren, R. J., Williams, W. L., Hardcastle, M. J., et al. 2016, ApJS, 223, 2

Venturi, T., Dallacasa, D., \& Stefanachi, F. 2004, A\&A, 422, 515

Wagner, A. Y., \& Bicknell, G. V. 2011, ApJ, 728, 29

Wagner, A. Y., Bicknell, G. V., \& Umemura, M. 2012, ApJ, 757, 136

Wegner, G., Haynes, M. P., \& Giovanelli, R. 1993, AJ, 105, 1251

White, R. L., \& Becker, R. H. 1992, ApJS, 79, 331

Williams, W. L., van Weeren, R. J., Röttgering, H. J. A., et al. 2016, MNRAS, 460, 2385

Worrall, D. M., \& Birkinshaw, M. 2006, in Physics of Active Galactic Nuclei at all Scales, ed. D. Alloin (Berlin: Springer Verlag), Lect. Notes Phys., 693, 39 Wu, Q. 2009, ApJ, 701, L95

Zhao, J.-H., Sumi, D. M., Burns, J. O., \& Duric, N. 1993, ApJ, 416, 51 